\title{
Über einige Aspekte der Arbeit von Peter Scholze
}

\author{
Torsten Wedhorn
}

\begin{abstract}
Im August 2018 erhielt Peter Scholze die Fieldsmedaille for transforming arithmetic algebraic geometry over $p$-adic fields through his introduction of perfectoid spaces, with application to Galois representations, and for the development of new cohomology theories.

Es ist wahrscheinlich nicht möglich, auf wenigen Seiten eine präzise Version oder auch nur einen einigermaßen vollständigen Eindruck der Vielzahl von bahnbrechenden Resultaten von Scholze zu geben. Selbst eine genaue Erklärung der obigen Kurzlaudatio der internationalen Mathematikunion würde sicherlich einen sehr viel längeren Text erfordern. Aber vielleicht ist es möglich, eine vage Idee des Begriffs eines perfektoiden Raumes zu geben und einen Eindruck zu vermitteln, warum diese Räume einen großen Teil der arithmetischen algebraischen Geometrie revolutioniert haben. Dies ist ein Versuch.
\end{abstract}

Zahlentheorie und $p$-adische Geometrie

In ihrer ursprünglichen Form beschäftigt sich Zahlentheorie mit der Lösung polynomieller Gleichungen in den ganzen Zahlen. Der „letzte Satz“ von Fermat etwa besagt, dass es keine von 0 verschiedenen ganzen Zahlen $x, y$, und $z$ gibt, so dass $x^{n}+y^{n}=z^{n}$, wobei $n \geq 3$ eine natürliche Zahl ist. Durch Division durch $z^{n}$ ist dies äquivalent zu der Aussage, dass die Gleichung $x^{n}+y^{n}=1$ keine rationalen Lösungen $x, y \in \mathbb{Q}$ mit $x, y \neq 0$ gibt.

Lösungen in den reellen Zahlen sind häufig einfacher zu finden. Die folgende Kurve zeigt die Menge der reellen Lösungen von $x^{3}+y^{3}=1$. Insbesondere ist es klar, dass es unendlich viele reelle Lösungen gibt, und es mag vielleicht etwas überraschend sein, dass kein Punkt auf der Kurve rationale Koordinaten hat $(\operatorname{außer}(0,1)$ und $(1,0))$, obwohl ja die rationalen Zahlen dicht in den reellen Zahlen liegen.

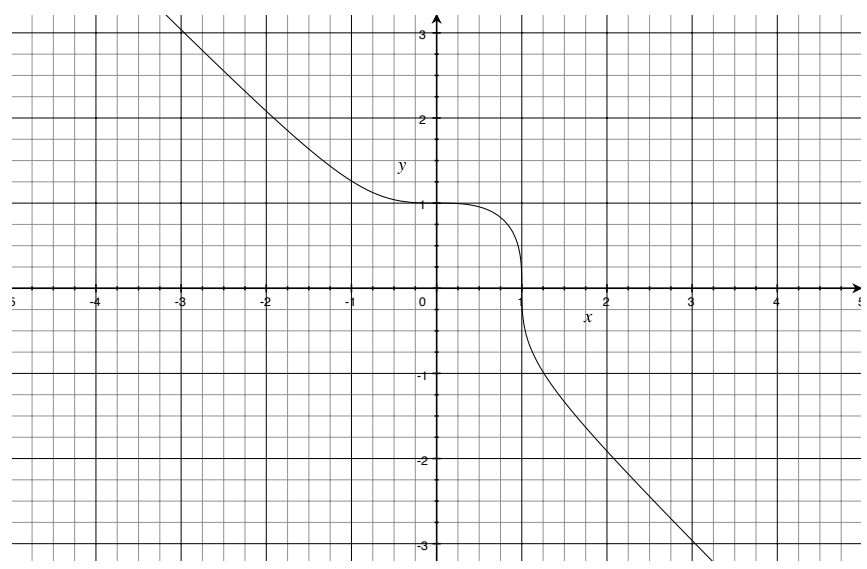

Nun entstehen die reellen Zahlen dadurch, dass man eine Abstandsfunktion, d.h. eine Metrik, auf den rationalen Zahlen definiert, wobei der Abstand zweier rationaler Zahlen $x$ und $y$ durch $|x-y|$ gegeben ist, und dann bezüglich dieser Metrik komplettiert, also „die Löcher bezüglich der Metrik auffüllt".

Diesen Prozess kann man genauso durchführen, wenn man den gewöhnlichen Absolutbetrag | | durch den $p$ adischen Absolutbetrag ||$_{p}$ ersetzt. Hier ist $p$ eine Primzahl, und $|x|_{p}$ ist umso kleiner, je häufiger $x$ von $p$ geteilt wird. Genauer schreibt man eine rationale Zahl $x \neq 0$ in der Form $x=p^{n} \frac{a}{b}$, wobei $n \in \mathbb{Z}$ ist und wobei $a$ und $b$ ganze Zahlen sind, die von $p$ nicht geteilt werden. Dann setzt man $|x|_{p}:=\alpha^{-n}$, wobei $\alpha>1$ eine fest gewählte reelle Zahl ist. Die Komplettierung von $\mathbb{Q}$ bezüglich der $p$-adischen Metrik ist der Körper der $p$-adischen Zahlen, bezeichnet mit $\mathbb{Q}_{p}$.

Die $p$-adische Metrik auf $\mathbb{Q}_{p}$ erlaubt es, von konvergenten Folgen und Reihen mit Koeffizienten in $\mathbb{Q}_{p}$ zu sprechen. Im gewissen Sinne ist hier die Konvergenz sogar einfacher, da eine Reihe $\sum_{n=1}^{\infty} x_{n}$ mit $x_{n} \in \mathbb{Q}_{p}$ genau dann in $\mathbb{Q}_{p}$ konvergiert, wenn $\left(x_{n}\right)_{n}$ eine Nullfolge ist. Dies ist eine Konsequenz aus der Tatsache, dass nicht nur die übliche Dreiecksungleichung $|x+y|_{p} \leq|x|_{p}+|y|_{p}$, sondern die stärkere nicht-archimedische Dreiecksungleichung

$$
|x+y|_{p} \leq \max \left\{|x|_{p},|y|_{p}\right\}
$$

gilt. Dies hat allerdings auch zur Folge, dass die Geometrie der $p$-adischen Zahlen eine Reihe gewöhnungsbedürftiger Aspekte besitzt, etwa dass sich zwei Kreisscheiben entweder gar nicht schneiden oder eine von ihnen bereits in der anderen enthalten sein muss.

Ähnlich wie nun (reell analytische oder komplexe) Mannigfaltigkeiten geometrische Gebilde sind, die lokal Null- 
fen sind, die aber über ihrem Analogon in Charakteristik $p$ bewiesen sind.

\section{Überlagerungen und Galoistheorie}

Ein klassischer Satz aus der Topologie klassifiziert für hinreichend zusammenhängende Räume $X$, z. B. zusammenhängende reelle oder komplexe Mannigfaltigkeiten, seine (unverzweigten) Überlagerungen, also Räume über $X$, die lokal von der Form $F \times X$ für einen nicht leeren diskreten Raum $F$ sind. Er besagt, dass es eine Bijektion zwischen Überlagerungen ${ }^{3}$ von $X$ und Untergruppen der Fundamentalgruppe von $X$ gibt. Dabei entsprechen endliche Überlagerungen den Untergruppen von endlichem Index.

Diese Aussage besitzt ein algebraisches Analogon in der algebraischen Geometrie, wo man ebenfalls den Begriff einer (algebraischen) Fundamentalgruppe definieren kann. Klassisch ist das Beispiel eines Körpers $k$, der aus Sicht der algebraischen Geometrie einem Punkt entspricht. ${ }^{4}$ „Punktierte zusammenhängende unverzweigte Überlagerungen solch eines Punktes" sind in dieser Sprache gerade durch endliche separable Teil-Erweiterungen eines gewählten algebraischen Abschlusses $\bar{k}$ gegeben. Die „Fundamentalgruppe von $k^{\prime \prime}$ ist die Galoisgruppe von $k$, d. h. die Automorphismengruppe von $\bar{k}$ über $k$. Und der Hauptsatz der Galoistheorie besagt gerade, dass die Menge solcher Erweiterungen gerade in Bijektion zu der Menge der Untergruppen von endlichen Index von der Galoisgruppe von $k$ ist. Er ist also ein genaues Analogon des obigen Satzes aus der Topologie. Insbesondere haben zwei Körper dieselbe „Überlagerungstheorie“, wenn sie dieselbe Galoisgruppe besitzen.

\section{Ein perfektoider Punkt}

Schon lange war bekannt, dass die Körper $\mathbb{Q}_{p}$ und $\mathbb{F}_{p}((t))$ viele formale Ähnlichkeiten besitzen, obwohl der eine die Charakteristik 0 und der andere die Charakteristik $p$ hat. So kann man durch $p$-adische Entwicklung die Elemente von $\mathbb{Q}_{p}$ ebenso als „Laurentreihen in $p$ mit Koeffizienten zwischen 0 und $p-1$ " betrachten

$$
\mathbb{Q}_{p}=\left\{\sum_{n \gg-\infty} a_{n} p^{n} ; a_{n} \in\{0,1, \ldots, p-1\}\right\}
$$

und die $p$-adische Bewertung ist wieder durch die „Nullstellenordnung der Reihe" gegeben. Des Weiteren besagt ein Satz von Fontaine und Wintenberger, dass die Galoisgruppen von $\mathbb{Q}_{p}$ und von $\mathbb{F}_{p}((t))$ sich mehr und mehr annähern, wenn man $p^{n}$-te Wurzeln der „Unbestimmten“ $p$ bzw. $t$ für $n \rightarrow \infty$ adjungiert. Im Limes stimmen die Galoisgruppen dann überein, und die beiden Körper $\mathbb{Q}_{p}\left(p^{1 / p^{\infty}}\right)$ und $\mathbb{F}_{p}\left(\left(t^{1 / p^{\infty}}\right)\right)$ besitzen daher dieselben endlichen unverzweigten Überlagerungen. Diese Konstruktion ist ein Spezialfall von perfektoiden Räumen und ihres Tilts.
Perfektoide Räume und der Tilt

Scholzes Interpretation des Resultats von Fontaine und Wintenberger ist die Konstruktion eines „Tiltingfunktors“ ()$^{b}$, der sogenannten perfektoiden Räumen in Charakteristik 0 einen perfektioden Raum in Charakteristik $p$ zuordnet. Für diesen Funktor gilt als 0-dimensionaler Spezialfall $\mathbb{Q}_{p}\left(p^{1 / p^{\infty}}\right)^{b}=\mathbb{F}_{p}\left(\left(t^{1 / p^{\infty}}\right)\right)$.

Ähnlich wie man $\mathbb{Q}_{p}\left(p^{1 / p^{\infty}}\right)$ als Limes der endlichen Körper-Erweiterungen $\mathbb{Q}_{p}\left(p^{1 / p^{n}}\right)$ erhält, kann man aus $p$ adischen Mannigfaltigkeiten perfektoide Räume der Charakteristik 0 erhalten, indem man aus lokalen Koordinatenfunktionen immer höhere $p^{n}$-te Wurzeln zieht. Man kann sich dies als eine Art „Fraktalisierung“ des $p$-adischen Raums vorstellen. Sobald man nun einen perfektoiden Raum $X$ hat, kann man seinen Tilt $X^{b}$ betrachten. Dies ist dann ein adischer Raum in Charakteristik $p$ und ist daher für viele Zwecke einfacher zu verstehen als die $p$-adische Mannigfaltigkeit, mit der man gestartet ist.

Des Weiteren zeigt Scholze, dass die Überlagerungstheorie eines perfektoiden Raums in Charakteristik 0 dieselbe ist wie die seines Tilts. Dies erlaubt es, in bisher nie dagewesener eleganter Weise von Räumen in Charakteristik $0 \mathrm{zu}$ solchen in Charakteristik $p$ zu wechseln.

In der Tat hat Scholze kürzlich einen direkten Weg aufgezeigt, einem $p$-adischen Raum ein geometrisches Objekt in Charakteristik $p$, einen sogenannten Diamanten, ohne den vorherigen Prozess der „Fraktalisierung“ zuzuordnen. Dies erlaubt es zum Beispiel, bislang nur vermutungsweise existierende $p$-adische Räume, sogenannte lokale ShimuraVarietäten, mit Methoden der Charakteristik $p$ zu konstruieren.

\section{Beispiele für Anwendungen}

Die Theorie der perfektoiden Räume hat sich in den vergangenen Jahren als extrem fruchtbar erwiesen. An dieser Stelle will ich nur zwei Anwendungen skizzieren, die die Worte „application to Galois representations“ und „development of new cohomology theories" aus der obigen Kurzlaudatio beleuchten sollen. Dies ist nur ein winziger Ausschnitt aus der Welt, die Scholzes Arbeiten der Mathematik eröffnet haben.

Die erste Anwendung ist Scholzes Konstruktion von Darstellungen von Galoisgruppen assoziiert zur Kohomologie von lokal symmetrischen Räumen für die allgemeine lineare Gruppe $\mathrm{GL}_{n}$ über total reellen Körpern oder mit komplexer Multiplikation. Die Existenz solcher Galoisdarstellungen wurde vor über 40 Jahren von Ash, Grunewald und anderen vermutet. Für Kohomologie mit rationalen Werten war dies kürzlich in einer Arbeit von Harris, Lan, Taylor und Thorne gezeigt worden. Das bemerkenswerte an Scholzes Resultat ist, dass er in der Lage ist, Galoisdarstellungen zur Kohomologie mit Werten in $\mathbb{Z} / p^{n} \mathbb{Z}$ zu assoziieren. Während rationale Kohomologieklassen recht selten sind, sind Torsionsklassen reichlich vorhanden. Das wichtigste Werkzeug ist wieder die Perfektoisierung, diesmal von Shimura- 


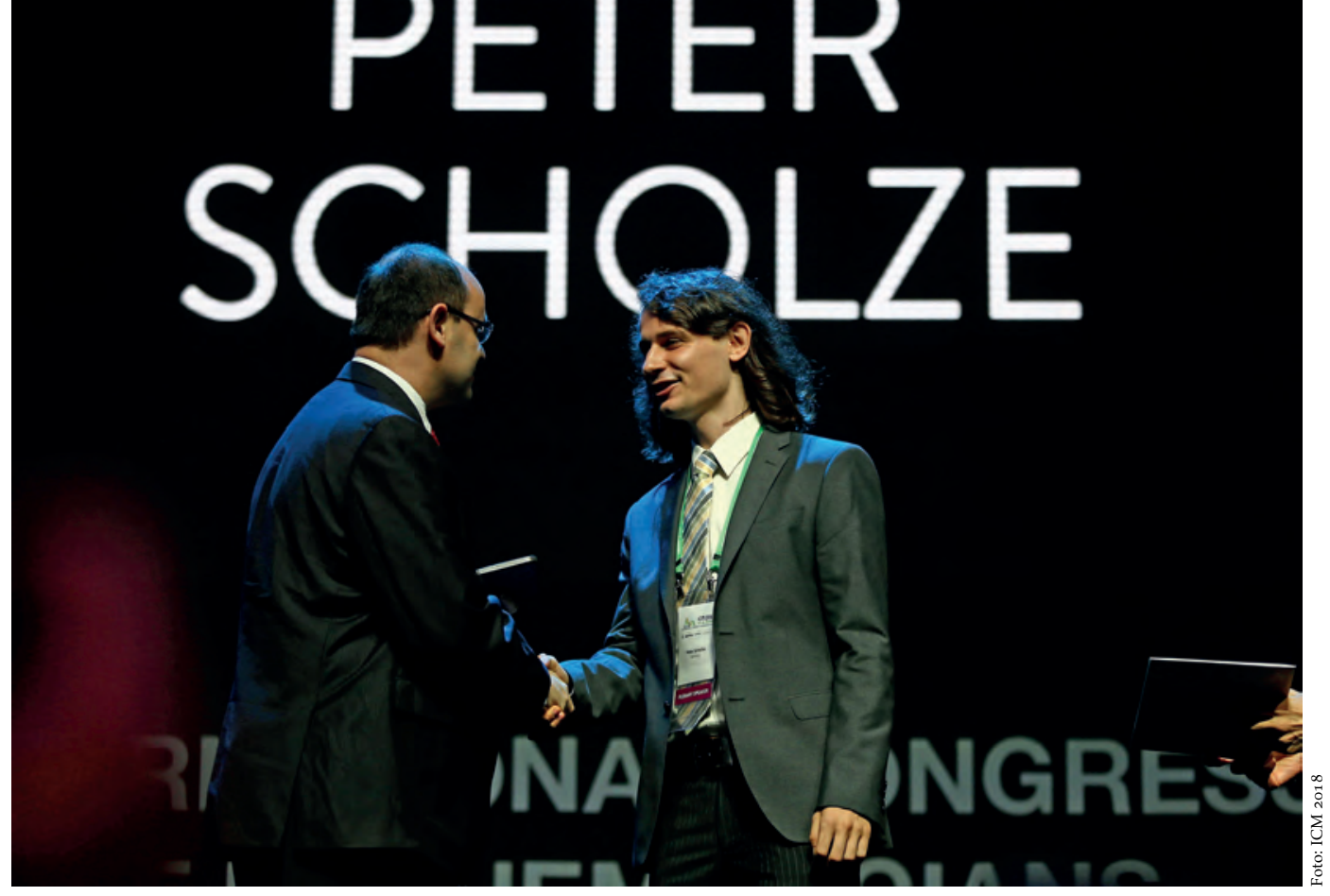

Peter Scholze wird auf dem ICM 2018 mit der Fieldsmedaille ausgezeichnet.

Varietäten, in deren Kohomologie man die Kohomologie der lokal symmetrischen Räume als Randbeitrag wiederfindet.

Eine zweite Anwendung ist die Konstruktion einer neuen Kohomologie-Theorie für kompakte glatte $p$-adische Räume mit guter Reduktion von Scholze in einer gemeinsamen Arbeit mit Bhatt und Morrow. Kohomologiegruppen sind wichtige algebraische Invarianten in der Geometrie. Ein Beispiel ist das Geschlecht einer kompakten Riemannschen Fläche, welches nichts anderes als der Rang einer gewissen Kohomologiegruppe ist.

Für $p$-adische Räume existieren mehrere solcher Kohomologietheorien. In ihrer Arbeit konstruieren Bhatt, Morrow und Scholze eine neue Theorie, so dass die wichtigsten bereits bekannten Theorien Spezialisierungen ihrer Theorie sind. Sie benutzen diese Technik unter anderem dazu, um Resultate über die Beziehungen von Torsionsklassen in den verschiedenen Kohomologien zueinander zu erhalten. Dies war ein zentrales ungelöstes Problem in der $p$-adischen Geometrie, das weit offen war seit den goer Jahren, in denen Fontaine, Messing, Faltings und Tsuji den torsionsfreien Anteil behandelt haben.

Dies ist nur ein kleiner Teil der Resultate, mit denen Scholze in den letzten Jahren Teile der Fundamente der arithme- tischen algebraischen Geometrie neu gestaltet hat. Seine Resultate strahlen dabei weit in andere Bereiche der Mathematik wie etwa die Topologie oder die Theorie der automorphen Formen hinein.

Bemerkenswert an Scholzes Ergebnissen ist die Einfachheit und Schönheit seiner Ideen. Zwar erfordert die Umsetzung dieser Ideen große technische Fähigkeiten, aber man hat bei seinen Arbeiten immer das Gefühl, dass er ganz natürliche Strukturen innerhalb der Mathematik freilegt, die - im Nachhinein - in ihrer Klarheit und Eleganz eigentlich immer schon offenkundig hätten sein sollen. Dank ihm ist die Mathematik noch einmal ein gutes Stück aufregender und faszinierender geworden.

\section{Anmerkungen}

1. Es war die Erkenntnis von John Tate in den 6oer Jahren, wie man "lokal“ in diesem Zusammenhang genau zu definieren hat.

2. Diese kann auch negativ sein, wenn $f$ einen Pol besitzt.

3. Genauer: von Isomorphieklassen punktierter, zusammenhängender Überlagerungen.

4. Da es natürlich viele verschiedene Körper gibt, gibt es also auch viele verschiedene Punkte in der algebraischen Geometrie.

Prof. Dr. Torsten Wedhorn, Technische Universität Darmstadt,

Fachbereich Mathematik, Schlossgartenstraße 7, 64289 Darmstadt wedhorn@mathematik.tu-darmstadt.de 